\title{
Selective Ozone Treatment of PDMS Printing Stamps for Selective Ag Metallization: A New Approach to Improving Resolution in Patterned Flexible/Stretchable Electronics
}

Gemma Francis $^{1}$, Bryan W. Stuart ${ }^{1}$, Hazel E. Assender ${ }^{*}$

${ }^{1}$ Department of Materials, University of Oxford, Parks Road, OX1 3PH

*Corresponding Author: Hazel E. Assender, hazel.assender@materials.ox.ac.uk

Keywords: Flexible electronics, Surface Energy, Thermal Evaporation, Patterning, Flexography, Ozone

\section{Abstract \\ Hypothesis}

Selective ozone treatment of Polydimethylsiloxane (PDMS) print-stamps may facilitate local de-wetting of Krytox ${ }^{\circledR} 1506$ oil; the resulting printed pattern can be used as a masking liquid during roll-to-roll vacuum-metallization, exemplified with Ag. This novel method may exploit high-throughput manufacture without chemical etchants or elevated temperatures for thin-film electronics.

\section{Experiments}

The mechanism for selective wetting arose from $\mathrm{O}_{3}$ treatment of PDMS through a shadowmask to vary surface-energy due to formation of polar silanol ( $\mathrm{Si}-\mathrm{OH})$ replacing surface methyl groups leading to contact angle reduction from $40^{\circ}-9^{\circ}$ for oil on PDMS. Oiled PDMS was (1) metalized itself and (2) used as a stamp to print onto polyethylene-terephthalate, consisting of oil pick-up/de-wetting/transfer-to-substrate/metallization.

\section{Findings}

Ag (520-568 nm) thick was deposited outside oiled regions, surpassing $\sim 20 \mu \mathrm{m}$ resolution of commercial printing. On metalized PDMS, minimum line widths were $2.6 \mu \mathrm{m}$ (with $10 \mu \mathrm{m}$ edge-grading from centrifugal oil spreading) or widths of $24 \mu \mathrm{m}$ (no Ag grading) following spin-coating/roll-coating oil respectively. The progressive effect of thinning oil via five successive stamp-to-substrate impressions, produced line widths of $14 \mu \mathrm{m}$ (with graded edge of $7.6 \mu \mathrm{m}$ via spreading from stamp-substrate compression). Developments may reduce reliance on laser engraving/photocuring, and could enhance micro-contact printing through liquid dynamics $v s$. topographical relief structures. 


\section{Introduction}

In recent years the demand for mass produced, flexible electronics has increased for nonintrusive, wearable and implantable devices. As a well-established technique, flexographic contact printing has been adapted for Roll-to-Roll (R2R) continuous manufacturing and is widely used to print conductive carbon- and silver-containing nano-particle inks in atmospheric processes for pressure sensors, solar cells, light emitting diodes and transistors [1] on flexible substrates including polyethylene terephthalate (PET), polyimide, or onto polydimethylsiloxane (PDMS) as the patterned substrate for production of stretchable electronics [2].

Whilst contact printing utilises an ink-covered, pre-patterned polymer relief based stamp to deposit ink through physical, stamp-to-substrate contact, non-contact methods such as inkjet rely on the relative movement of ink-ejecting nozzles and are significantly hindered by the "The Coffee Ring Effect". Printing inks are limited in minimum thickness due to surface tension, creating significant topographic steps that are un-desirable for subsequent device layers, and also in their ability to match the bulk electronic properties of metals and semiconductors. A further commercial obstacle is difficulty/expense in synthesising many conductive metals into stable nanoparticle inks, thus a printing technique utilising a pure metal, is desirable [3]. Consequently, a technique known as "selective metallization" was patented in the 1970s which enables patterning of metals from angstrom to nanometre thickness by thermally evaporating a desired metal towards a low vapour pressure Krytox ${ }^{\circledR}$ or Fomblin ${ }^{\circledR}$ oil mask, previously transferred to the substrate by a relief based patterned stamp, to selectively inhibit the deposition $[4,5]$. By shifting the technology from nanoparticle inks to bulk metal layers the solvent drying step is eliminated [6], defects are minimised, thickness uniformity improves and surface roughness may be reduced (previously limited by nanoparticle size) in deposited layers.

Going one step further, the ability to selectively deposit semiconductors, oxides, and polymers in vacuum environments will enable patterning of the vast range of circuitry components. Flexography as a R2R ink technique has reported resolutions of 20-100 $\mu \mathrm{m}$ [7] whilst lines of $1 \mu \mathrm{m}$ have been printed using micro-flexography [8].

The current bottle-neck in the resolution of relief based stamps is the effect of distortion of the profiled pattern printhead and liquid spreading during stamp-substrate compression as opposed to desirable congruent transfer, additionally the current reliance on manufactured moulds for curable photopolymers and laser engraving equipment for nitrile rubber sleeves can limit the 
resolution of the print plate features [9]. Commercially, flexo print resolutions have been limited to $\sim 30 \mu \mathrm{m}$ where minimum laser spot sizes of about $11 \mu \mathrm{m}$ and combined effects of liquid spreading restrict high resolution [10]. Researchers at the Massachusetts Institute of Technology (MIT) have developed patterned stamps using aligned carbon nanotubes (CNTs) to control/minimise ink spreading. CNTs were grown on a silicon substrate and surfacemodified with a CVD-deposited polymer. The pattern, which is formed by lithographically patterned catalyst for the local growth of the CNTs, allowed printed line widths of $3 \mu \mathrm{m}$. Although the stamps satisfactorily showed good compressive recovery and were tested for 100 print cycles, they are yet to be developed to be $\mathrm{R} 2 \mathrm{R}$ compatible which would require a change in the CNT growth method which currently utilizes rigid silicon [11].

Along similar lines, the authors here have investigated the effects of ultra-violet generated ozone irradiation for altering the surface-energy of PDMS (used both as a printing stamp onto flexible PET and as a stretchable substrate itself).

PDMS has been widely researched for use in optical and microfluidic devices $[12,13]$ and is the most widely used material for micro-contact printing stamps due to its conformity, able to print features down to $1 \mu \mathrm{m}$ [14]; a key advantage over commercially used photopolymer which is limited to $20-30 \mu \mathrm{m}$ line widths from light scattering effects during the plate exposure [15] and over laser engraved nitrile sleeves which have plate feature resolutions down to $10 \mu \mathrm{m}$. PDMS stamps have been shown to print features down to $1 \mu \mathrm{m}$ by precision control of stamp to substrate compression shown by Deng et al., far surpassing the current limit of photopolymer or nitrile plates [8]. Cosnahan et al. used profiled PDMS in R2R selective metallization of $\mathrm{Al}$ electrodes to achieve $150 \mu \mathrm{m}$ resolutions, concluding that print fidelity and resolution depends not primarily on the vaporization behaviour, but on the quality of the oil printing, including spreading effects due to distortions of the profiled print plate [6]. The deformation of the topographic features due to the elastomeric properties of the print plate material is a common issue in achieving lower resolutions [16]. Hence the novelty here is a method of creating a pattern that is less or not reliant on topographic precision, as it can be based on planar print plates, which would be a significant manufacturing step-change for printing abilities of PDMS in both micro-contact and R2R flexographic printing on the lab and high throughput scales respectively.

Current research on ozone modification of PDMS is related to polar liquids in microfluidic devices [13, 17-19]. Ozone has been shown to modify the surface of PDMS to affect the wettability of water from $96.7^{\circ}$ to $7.5^{\circ}$. In the work presented here selective ozone $\left(\mathrm{O}_{3}\right)$ 
irradiation will be shown as a viable method to manipulate Krytox ${ }^{\circledR} 1506$ masking oil in selective locations, to control liquid spreading beyond intended resolutions, thus opening up the possibility to replace a profiled laser-scribed print plate with one that can be easily formed from a simple ozone treatment through a shadow mask for subsequent use in roll-to-roll printing from a surface-patterned planar print plate. 


\section{Methodology}

\section{PDMS Manufacture}

PDMS was manufactured using Sylgard ${ }^{\circledR} 184$ (Dow Corning) consisting of a silicone elastomer base and cross-linking agent. The two components were mixed for $10 \mathrm{~min}$ in a 10:1 and degassed at $1 \times 10^{-1} \mathrm{mbar}$ for $45 \mathrm{~min}$. The degassed mixture was poured into a glass petri dish (110 $\mathrm{mm}$ diameter) to act as a mould for planer stamps or an $\mathrm{Al}$ frame mounted on glass (the frame measured $150 \times 50 \times 5 \mathrm{~mm}$ and acted as a print plate mould for R2R processing). The mixture was cured for $1 \mathrm{~h}$ at $100{ }^{\circ} \mathrm{C}$ in an oven in atmospheric conditions.

\section{Ozone Treatment and Masking}

PDMS surfaces were cleaned by sonicating in ethanol for 10 min then ozone treated 3 days after curing. Ma et al. investigated the varied effect of ozone treatment with curing time showing no change following 3 days [19]. A Novascan PSD Series Digital UV Ozone System was used to irradiate samples at a UV bulb-to-sample height of $5 \mathrm{~mm}$. $30 \mu \mathrm{m}$ thick shadow masks (typically used for evaporation of field effect transistor source-drain contacts) with feature gaps of 30, 40, 50, 60 and $80 \mu \mathrm{m}$ were acquired from Ossila Ltd (see figure 1). For creating patterns, masks were placed on PDMS throughout the duration of ozone treatment for $30 \mathrm{~min}-4 \mathrm{~h}$. Following ozone treatment shadow masks were peeled away from the PDMS surface, suggesting good surface-surface contact and limited shadowing effects. The maximum applied power density was $28-32 \mathrm{~W} / \mathrm{cm}^{3}$ at a wavelength of $253.7 \mathrm{~nm}$. Relative power densities at all other emitted wavelengths can be found in supplementary data.

\section{Krytox $^{\circledR} 1506$ Oil Application (Spin Coating or R2R)}

$200 \mu \mathrm{L}$ of oil was deposited using a Laurell [Model WS-650SZ-6NPP/LITE] spin coater using speeds from 2000-6000 rpm and varying times of 40-180 sec. Oil thickness was approximated by measuring weight change pre- and post- spin coating via a Mettler Toledo Micro Excellence Plus XP Analytical Balance accurate to $0.01 \mathrm{mg}$. PDMS stamps were cut to an approx. area of $2.5 \times 2.0 \pm 0.3 \mathrm{~cm}$ and stamp to PET substrate compression force was via the stamps own weight, therefore applying 0.5 mbar pressure. Sample weight was calculated using the theoretical density for PDMS of $965 \mathrm{~kg} / \mathrm{m}^{3}$.

To mimic a R2R flexographic printing process, a custom flexography system reported in Ref [20] was tested. Oil was picked up by an anilox roller, wiped with a doctor blade assembly and rolled against a PDMS print plate (fixed to the plate roller) at constant impression pressure of $1 \mathrm{mbar}$ at a roll speed $\sim 5.5 \pm 0.5 \mathrm{~m} \mathrm{~min}^{-1}$.

\section{Metal Deposition (Thermal Evaporation and Thickness)}


An Edwards E306A Vacuum Thermal Evaporator was used to deposit a thin layer of silver (99.9\% Purity, acquired from Argex Ltd. as $1 \mathrm{~mm}$ wire) onto the patterned PDMS or $12 \mu \mathrm{m}$ thick PET samples. Silver was evaporated from a tungsten boat (Buhler $\mathrm{GmbH}$ ). Silver was used for this study, but other evaporated metals, such as Al, are also possible. A base vacuum pressure of $9 \times 10^{-6}$ mbar was achieved following consecutive rotary (10 min) and diffusion pumping (30 min) stages. Silver thickness was kept consistent between batches using a quartz crystal micro-balance and was subsequently measured using a Veeco DekTak stylus profilometer on partially masked and coated silicon wafers.

\section{Contact Angle}

At least $n=5$ individual droplets of oil were syringed onto the PDMS 10 min after completing ozone treatment using a 0.26 diameter syringe and imaged via a Dino-Lite Digital Microscope. Images were taken $10 \mathrm{sec}$ after the droplet was placed to assess the static contact angle and to reduce the error caused by time dependent liquid spreading. Image-J was used to analyse contact angle from images via the plug-in "Drop Snake" [21]. Drop Snake is reliant on user plotted points of the air/curve boundary and drop profile, and the commensurate error is represented here by the average and standard error of $n=5$ droplets (left and right angle), $n=2$ independent images whilst undertaking the repeated analyses five independent times.

\section{Surface Roughness (confocal profilometry and AFM)}

A NanoFocus ${ }^{\circledR}$ AG, 2003 confocal white light source microscope was used to measure the surface macro-roughness. An Olympus UMPLFL 50X objective was fitted with a numerical aperture of 0.8 and working distance of $0.66 \mathrm{~mm}$. All samples were analysed using the software $\mu \operatorname{surf}^{\circledR}$ on image profiles to obtain Sa values. Four 320 × $320 \mu \mathrm{m}$ areas of each sample were analysed to determine whether the sample surface was uniform over ozone treated and nontreated areas. Similarly, nano-roughness was analysed in tapping mode using an AFM (JEOL JSTM-4200D) with NCHV-A, Bruker Ltd. tips. Each measurement represents four randomly selected locations of $0.5 \times 0.5 \mu \mathrm{m}^{2}$.

\section{Fourier Transform Infrared Spectroscopy (FTIR)}

A Varian Excalibur FTS 3500 FTIR with an Attenuated Total Reflection [ATR] attachment containing a diamond crystal and $\mathrm{ZnSe}$ lens was used measure absorption spectra in the wavenumber range $500-4000 \mathrm{~cm}^{-1}$ with a resolution of $4 \mathrm{~cm}^{-1}$.

\section{Thickness Measurement}

Ag coating thickness on PDMS was measured by stylus profilometry using a Bruker Dektak $6 \mathrm{M}$. Thicknesses represent the average of $n=6$ edges between coated and masked regions. Prior 
to measurement, any excess in oil remaining after metallization was removed by wiping with isopropanol.

\section{Surface Images}

Micrographs were acquired using a Leica M165C light microscope. Scanning Electron Microscopy (SEM) was undertaken via a Hitachi TM3000 at a working distance of $10 \mathrm{~mm}$ and beam voltage of $15 \mathrm{kV}$.

\section{Results and Discussion}

PDMS has been frequently used for microfluidic channels and for micro-contact printing, benefitting from submicron topographical conformity [14]. Here we show that ozone-treated PDMS may be used independently to produce stamps by facilitating localised de-wetting via altering surface chemistry. This could be applicable, for example, to R2R selective metallization for vapour deposited patterned electronics to overcome challenges associated with manufacture of Ink based electrodes in flexible electronics. Whilst electronic performance of deposited metals has not been the focus of this paper a recent publication by Stuart et al. [20] shows the upscale potential of krytox oil masking for producing $\mathrm{Al} / \mathrm{Cu} / \mathrm{Ag}$ electrodes by R2R single step selective metallization where $4 \times 6 \mathrm{~mm}$ metallic electrodes were deposited at $25 \mathrm{~m} \mathrm{~min}^{-1}$ using conventional nitrile and photopolymer stamps. Electrodes showed enhanced conductivity as compared to more conventional atmospheric ink based technologies found in literature. The process schematic shown in Figure 1 illustrates the development of the manufacturing process with multiple final products culminating in a masking technology usable in R2R manufacturing, from initial oil application on PDMS, to mask transfer onto PET. The resulting metallized patterns are shown in Figure 1: patterned PDMS elastomer substrate/print stamp with oil applied by spin coating (Sample 1) or by R2R oil transfer (Sample 2), and the final stage of R2R patterning onto PET by PDMS stamp-to-PET contact (Sample 3). In Step 1, PDMS was selectively ozone treated through a patterned shadow mask. The influence of $\mathrm{O}_{3}$ treatment to facilitate selective wetting was tested by spin coating in step $2 a$. Ultimately masks produced using oiled PDMS (by spin coating) in Step $2 a$ and on oiled PDMS (by R2R oil transfer) were shown to be effective for masking selectively treated areas for subsequent Ag thermal evaporation (Step 3a)(Sample 2). Secondly, the ability to use treated and oiled PDMS as a R2R stamp on PET was also tested and showed promise in Step $3 b$ (Sample 3). 


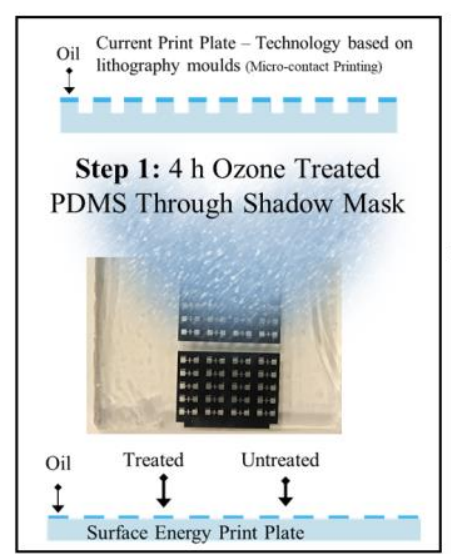

Sample 1: Ag Coated PDMS (Spin Coated Oil)

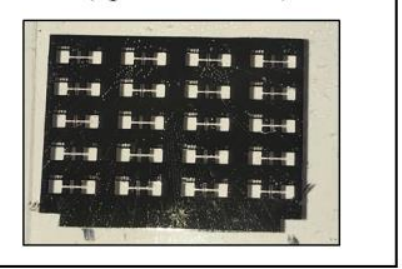

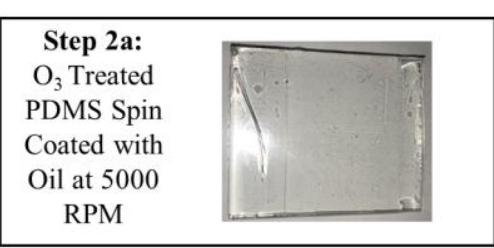

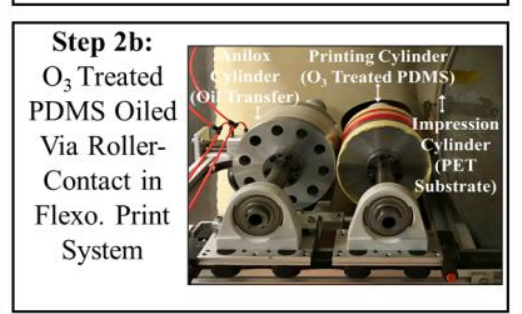

Sample 2: Ag Coated PDMS (R2R Coated Oil)

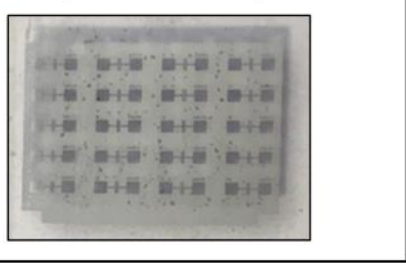

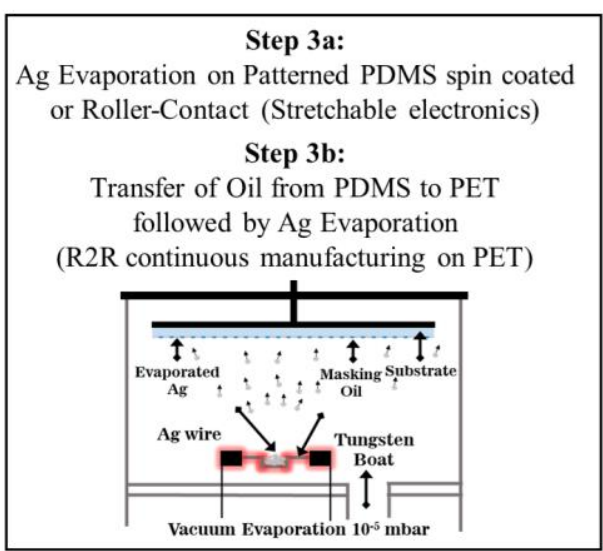

Sample 3: Coated PET

(R2R Oiled on PDMS + Stamp on PET )

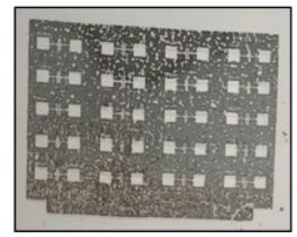

Figure 1: Selective ozone $\left(\mathrm{O}_{3}\right)$ treatment of PDMS was shown to induce a sufficient change in surface energy to constrain masking oil within treated areas. The process schematic demonstrates possible manufacturing iterations for production of patterned Ag on either PDMS (for stretchable electronics) or PET (for roll-to-roll-processed flexible electronics). In each case, a lab scale process is demonstrated by spin coating of masking oil on PDMS. Commercial scale early potential is shown by oil transfer from an anilox roller onto patterned PDMS and then, when desired, onto PET in a process used for R2R manufacture. Samples 1-3 show each stage to understand any reduction in conformity/resolution with each successive transfer stage.

\section{Contact Angle and Structural Variation (FTIR)}

Untreated PDMS is hydrophobic (water contact angle of $105^{\circ}$ ), surface modification by $\mathrm{O}_{3}$ irradiation reduces the contact angle linearly with exposure time to $15^{\circ}$ by $180 \mathrm{~min}$ as shown in Figure $2 A$. 

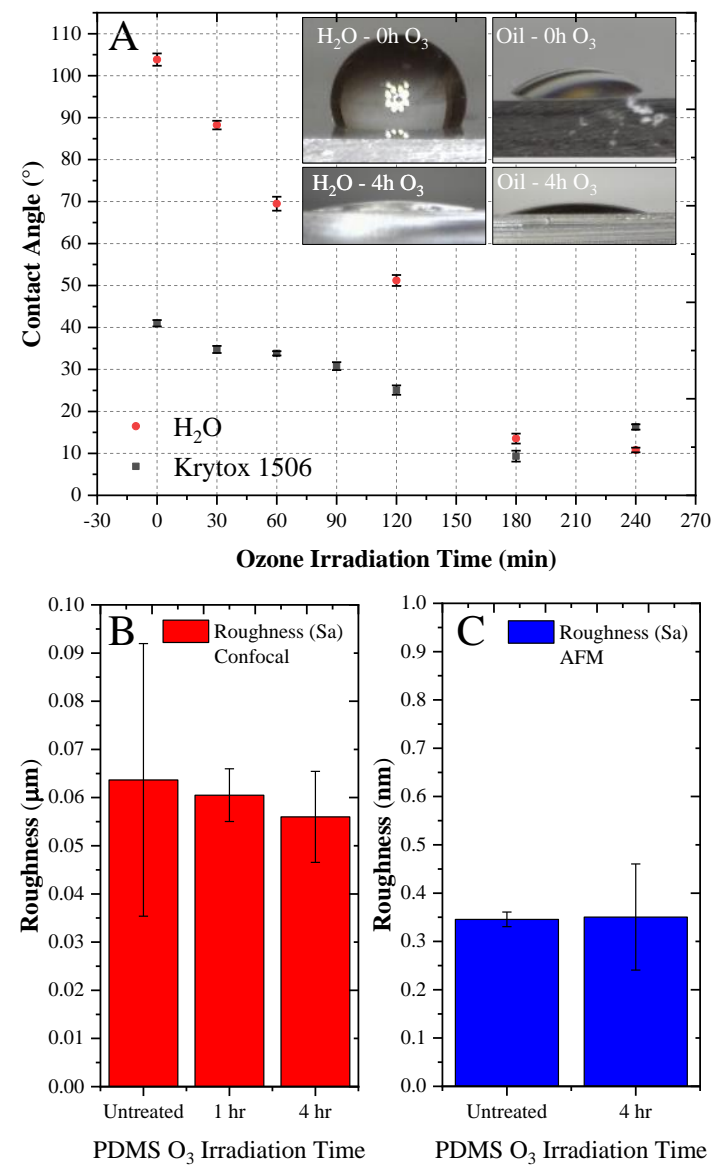

Figure 2: (A) Contact angle of $\mathrm{H}_{2} \mathrm{O}$ and Krytox ${ }^{\circledR} 1506$ oil on PDMS surface to observe wettability with ozone irradiation time up to $240 \mathrm{~min}$. (B) and (C) Macroscale surface roughness by confocal microscopy and nano-roughness by AFM respectively showing no significant change due to ozone irradiation. Error bars indicate the standard error from four $0.5 \times 0.5 \mu \mathrm{m}^{2}$ measurements.

The mechanism of $\mathrm{O}_{3}$ production in the ozone source is the reaction of molecular oxygen with $\mathrm{O}_{2}$ which had been dissociated into free oxygen radicals by ultra-violet (UV) radiation. $\mathrm{O}_{3}$ reacts with the PDMS surface via the substitution of the nonpolar methyl group in $\mathrm{Si}-\mathrm{CH}_{3}$ with the polar hydroxyl group to form $\mathrm{Si}-\mathrm{OH}$ silanols at the surface thereby making the surface increasingly hydrophilic. Untreated PDMS is predominantly dispersive 0.019 vs. polar 0.008 $\mathrm{J} / \mathrm{m}^{2}$ [22], attributed to the prevalence of $\mathrm{Si}-\mathrm{CH}_{3}$ bonds . The FTIR spectra presented in Figure 3 show the emergence of Si-OH groups, with absorption at $\sim 900$ and $\sim 3300 \mathrm{~cm}^{-1}$, present after 1 and $4 \mathrm{~h}$ of $\mathrm{O}_{3}$ treatment and confirmed the substitution with silanol of the methyl groups by the relative reduction of infrared absorption associated with the $\mathrm{Si}-\mathrm{CH}_{3}$, absorbing at $\sim 1260$ and $\sim 2950 \mathrm{~cm}^{-1}$ [23]. Brunauer et al. reported the relatively high surface energy of pure silanol in hydrous amorphous silica to be $0.129 \mathrm{~J} / \mathrm{m}^{2}$, greater than that of water $\left(0.072 \mathrm{~J} / \mathrm{m}^{2}\right)$ [24]. In comparison, untreated PDMS values are considerably lower, ranging from 0.016 to $0.024 \mathrm{~J} / \mathrm{m}^{2}$ to suggest that a conversion of the structure to silanol groups would in fact lead to the observed 
hydrophilic transformation; an effect widely confirmed in literature [19]. In support of this Efimenko et al. found that surface energy of $\mathrm{O}_{3}$ treated PDMS increased from $\sim 0.016$ to $\sim 0.070$ $\mathrm{J} / \mathrm{m}^{2}$ (similar to water), reaching a maximum by $60 \mathrm{~min}$ irradiation time [24].

The interaction between solid PDMS and liquid perflouropolyether (PFPE Krytox ${ }^{\circledR}$ oil) showed a similarly linear reduction in contact angle with treatment time, in this case from $40^{\circ}$ to $9^{\circ}$ (Figure 3) for untreated and $\mathrm{O}_{3}$ treated PDMS respectively. The initial contact angle was lower $\left(40^{\circ}\right)$ than that for water, explained by van der Waals liquid-surface interactions, so there is greater liquid wetting of the oil on PDMS. Following $\mathrm{O}_{3}$ treatment, the Krytox ${ }^{\circledR}$ oil contact angle reduced to $9^{\circ} \pm 1$, attributed to the increased surface energy of PDMS of $0.070 \mathrm{~J} / \mathrm{m}^{2}$ as reported by Efimenko et al [24]. Krytox is neither polar nor dispersive, as such, no innate attractions between $\mathrm{C}-\mathrm{F}$ and $\mathrm{Si}-\mathrm{OH}$ groups have been suggested and wettability may be dominated by surface energies/tensions. A distinct challenge associated with PDMS treatment is hydrophobic recovery over a number of days, caused by outwards diffusion of oligomers in the untreated subsurface ascribed to the extraordinarily low glass transition $\left(T_{g}\right)$ of PDMS $\left(\approx-120^{\circ} \mathrm{C}\right)$ permitting molecular mobility [13]. It should be noted that this recovery was not observed while sample remained coated with Krytox ${ }^{\circledR}$ oil over three days.

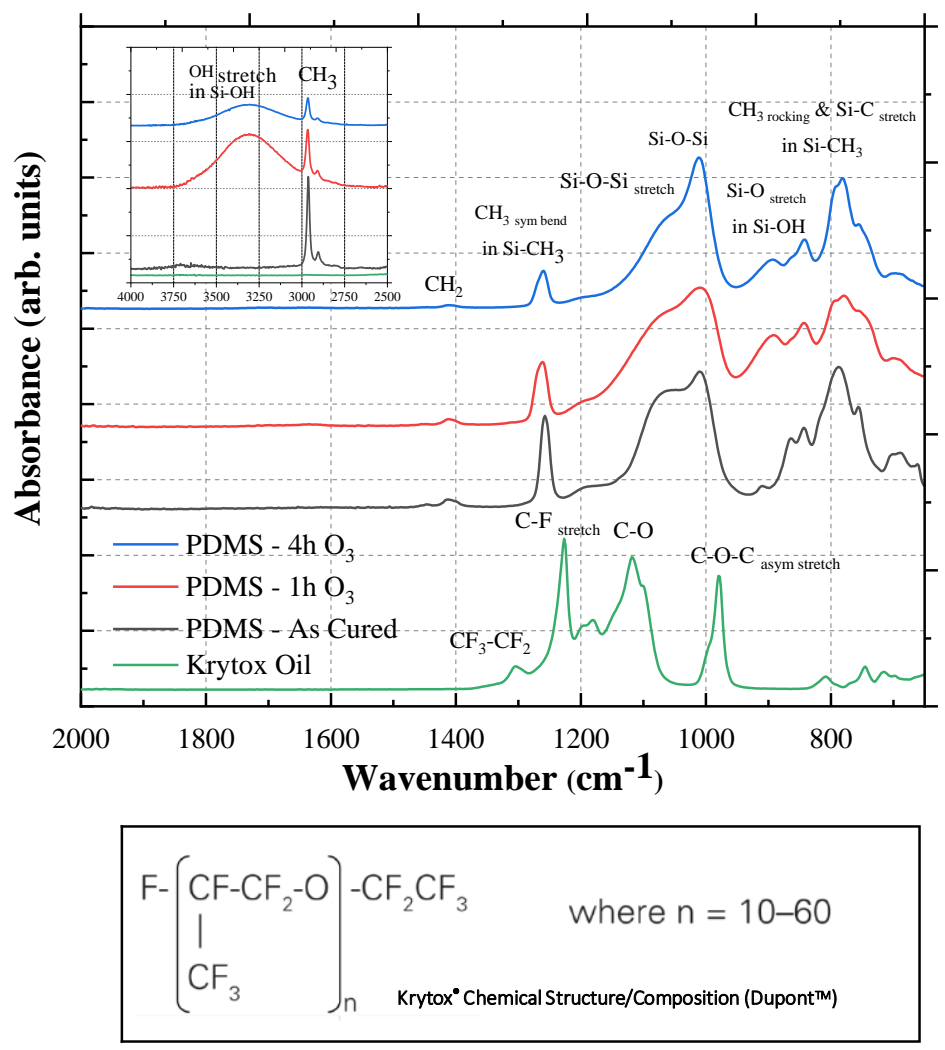

Figure 3: FTIR spectra of Krytox 1506 Oil showing characteristic structural bonds of the fluorocarbon structure, $C-F, C-O$ bonding and spectra of untreated and $\mathrm{O}_{3}$ irradiated PDMS 
[23]. Transformation of dispersive $\mathrm{CH}_{3}$ methyl groups in untreated PDMS were replaced by polar $\mathrm{OH}$ hydroxyl groups to cause the increased wettability of $\mathrm{H}_{2} \mathrm{O}$ on treated PDMS. The Krytox structure consisting of fluorocarbon bonds [25].

\section{Topography (confocal profilometry and AFM)}

Roughness measurements by both confocal microscopy and AFM showed roughness scales over 100 x $100 \mu \mathrm{m}$ (typical size of topographical relief features) and 500 x $500 \mathrm{~nm}$ scale (able to influence liquid wetting) respectively. The mean surface roughness for PDMS by confocal microscopy was $63 \mathrm{~nm}$ (Figure $2 B$ ) before treatment, with the mean reducing to $56 \mathrm{~nm}$ after four hours of treatment, but comfortably remaining within the spread of values. AFM of untreated and $4 \mathrm{~h} \mathrm{O}_{3}$-treated PDMS both exhibited identical surface roughness with a mean value of $0.35 \mathrm{~nm}$ (Figure 2C), confirming that ozone irradiation had no significant effect on surface roughness. Generally, as observed by Wenzel, an increase in surface roughness of $100 \mathrm{~s}$ of $\mu \mathrm{m}$ would increase surface de-wetting [26]. Since neither roughness variation nor de-wetting were observed, it suggests that van der Waals forces and polar attractions dominated liquid wetting on the nanoscale for Krytox $^{\circledR}$ oil and water respectively and additionally no topographical alterations at the scale of 100s of $\mu \mathrm{m}$ (able to increase wettability or within the order of magnitude of print resolutions (30-80 $\mu \mathrm{m}))$ were present.

\section{Spin Coating and Krytox ${ }^{\circledR}$ Oil Distribution}

Krytox $^{\circledR}$ oil was distributed over the PDMS surface by spin coating to observe the effect of increased wettability by ozone treatment on oil thickness. The mechanisms for the oil masking during vapour deposition suggested in literature are: i) The masking oil reaches its vaporization temperature following radiant heating from the evaporation source, generating a vapour cloud that repels the incoming condensate [6]; ii) The condensation energy of the depositing metal leads to oil ablation, taking the incoming metal away. The intention here was to achieve an oil thickness following ozone treatment which mimicked optimal oil transfer during $R 2 R$ manufacturing from anilox cylinder to print cylinder (shown in Figure 1, Step 2b). In two publications demonstrating oil masking with vapour deposition using Krytox ${ }^{\circledR} 1506$, firstly Cosnahan et al. approximated that the theoretical oil mask thickness to achieve optimal patterning (situation where the mask would be sufficiently thick to repel condensing metal throughout the deposition time) was $\sim 1.6 \pm 0.3 \mu \mathrm{m}$ when operating an oil patterned $\mathrm{R} 2 \mathrm{R}$ substrate at $2.4 \mathrm{~m} \mathrm{~min}^{-1}$ and depositing 30-50 nm thick electrodes [6]. Secondly Stuart et al. deposited $37 \mathrm{~nm} \mathrm{Al}$ electrodes with oil thickness $204 \pm 75 \mathrm{~nm}$ when operating at $25 \mathrm{~m} \mathrm{~min}^{-1}$. Therefore, as the Ag metallization here was undertaken on a static oil-masked sample, spin 
coating conditions were derived on unpatterned PDMS, to give an oil thickness (determined by weighing samples of known area) within the range from $0.2-1.6 \mu \mathrm{m}$. The oil thinning achievable by spin coating was ultimately limited by fluid viscosity and oil/surface interactions between oil and PDMS [20].

As expected, increasing spin coat speed from 2000-5000 RPM caused an apparent reduction in oil thickness (Figure 4A). Oil thickness was lower for the untreated specimens, as the attraction between PDMS and oil was lower $\left(40^{\circ}\right.$ contact angle as in untreated PDMS). In all cases, extending spin duration from 40 to $180 \mathrm{sec}$ showed a significant reduction in oil thickness, and a study of spin time at a spin speed of 5000 RPM (Figure 4B) indicated that the thickness plateaued between 180-240 sec. An oil thickness of $1.24 \mu \mathrm{m}$, within the desired range, was achieved from spin coating at 5000 RPM for $180 \mathrm{sec}$ on the $4 \mathrm{~h} \mathrm{O}_{3}$ treated PDMS surface.
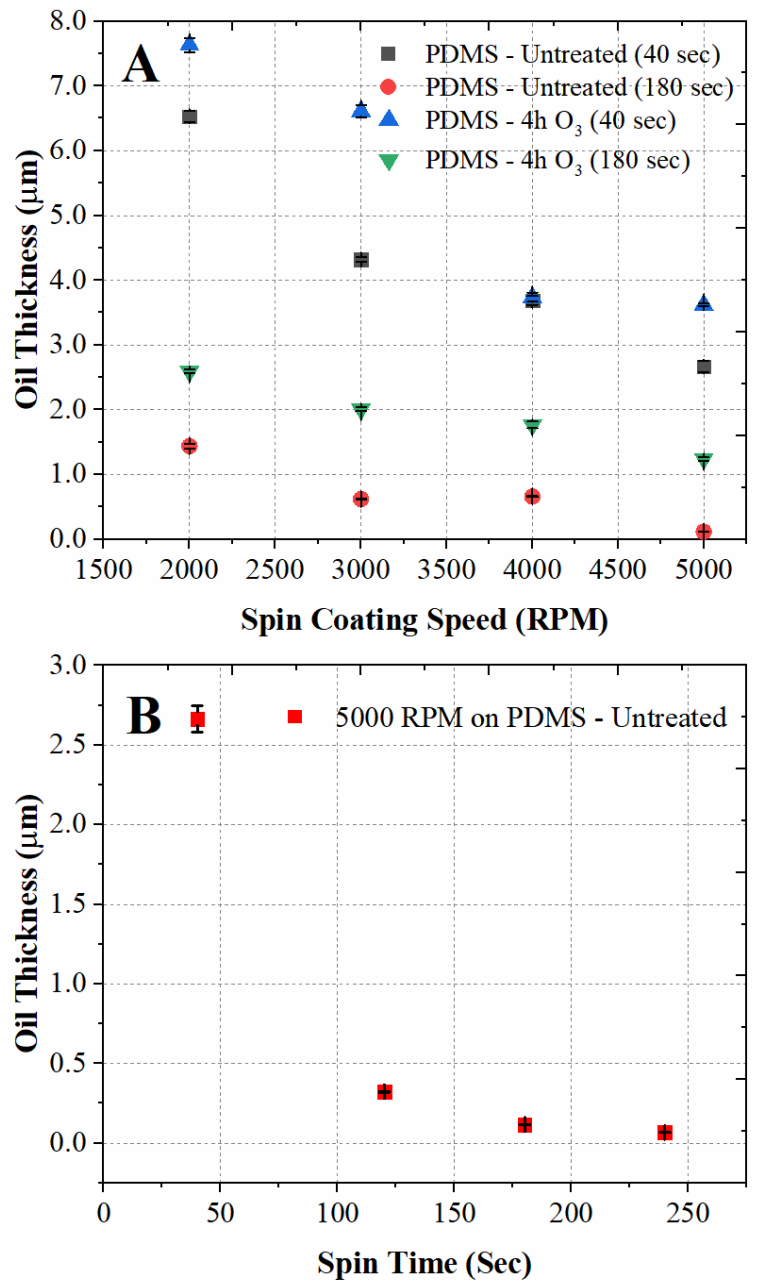

Figure 4: Krytox 1506 masking oil was spin coated on untreated and $4 \mathrm{~h} \mathrm{O}_{3} \mathrm{PDMS}$ to thin the oil layer down to mimic the quantity transferred during $R 2 R$ anilox to plate cylinder operation. Spin coating additionally forced the separation of oil to observe selective dewetting. A linear spin speed vs. oil thickness and a hyperbolic spin time vs. oil thickness were observed. 


\section{Masking Quality}

Now that the ability to change the surface energy of the PDMS with ozone without significant modification to surface topography is established and quantified, and the thickness of oil established for spin coating under different conditions, the patterning of the oil, and subsequent patterning of an evaporated metal can be tested. The expectation would be that, within an appropriate range of oil thickness and local treated/untreated area ratio, if there is sufficient difference in contact angle, the oil will dewet on the surface such that the boundary of the droplets would follow the boundary of the ozone-treated areas, to minimise interfacial energy, such that the oil would be confined to the ozone-treated regions. Metallization resolution was observed at each successive print stage illustrated in Figure 1 on both PDMS and PET. It should be noted here that the ozone-treated regions, defined by the gaps in the original shadow mask, are those with the lowest contact angle with the oil, and hence are the regions onto which the oil may become confined on preferential wetting. Thus, the pattern of metal, the negative of this, will be the 'positive' pattern of the original shadow mask. As shown in Figure 5A, nominal feature separations on the mask varied from 30 to $80 \pm 7 \mu \mathrm{m}$ as quoted by the manufacturer [27]. The shadow mask's feature gap was sharply defined, termed here "sharp distance" for the purpose of comparative measurements.

\section{Sample 1 (4h $\mathrm{O}_{3}$ treated PDMS 5000 RPM oil Spin Coated, Ag Coated)}

Figure $5 B$ successfully showed that selective de-wetting between $\mathrm{O}_{3}$ treated and untreated regions was achieved by spin coating (a relatively harsh separation condition with high radial forces, compared to eventual R2R operation). Ag coating thickness was measured as $519 \pm 17$ $\mathrm{nm}$. As intended, Ag deposition did not occur within the ozone-treated regions. Shortcomings were observed, specifically i) stray oil spots and oil splatter were randomly present forming droplets that masked the metallization, ii) imaging of the feature gap revealed a "graded distance" of Ag encroaching inwards to narrow the line width, which in the most narrow 30 $\mu \mathrm{m}$ nominal separation led to no "clearance gap" present (Figure 5B). Grading may have been caused by i) oil advancing beyond the masked boundary from centrifugal spreading whilst spin coating and ii) as oil thins at the droplet edge, over-metallization may have led to shrinkage of the oil droplet and graded edges as illustrated in Figure 6A. SEM micrographs of the print showed remaining oil within treated channels after the metal deposition (Figure 6B) to suggest that post process oil removal is necessary and deposition could continue until all oil evaporates from the channels. The measured graded distance was $\sim 9.5-11.6 \mu \mathrm{m}$, seemingly independent of nominal gap size of $60-80 \mu \mathrm{m}$ and graded Ag intersected for 40 and $30 \mu \mathrm{m}$ nominal gaps 
whilst clearance distances ranged from 2.6 to $41.4 \mu \mathrm{m}$ for shadow mask widths of 45.5 and $76.1 \mu \mathrm{m}$ respectively (Figure $5 A, B$ and $7 A$ ). An image showing the smallest clearance distance of $2.6 \mu \mathrm{m}$ for nominal/measured channel width of $50 / 45.5 \mu \mathrm{m}$ can be found Figure 8 . Notably, oil spreading/grading led to significant narrowing of the Ag line clearance distance with respect to the $\mathrm{O}_{3}$ treated area, where shrinkage ranged from $46-94 \%$ and whilst this could be perceived as a challenge, if predictable could be a route to making micro- or nano-features as shown by the $2.6 \mu \mathrm{m} \mathrm{Ag}$ channel.

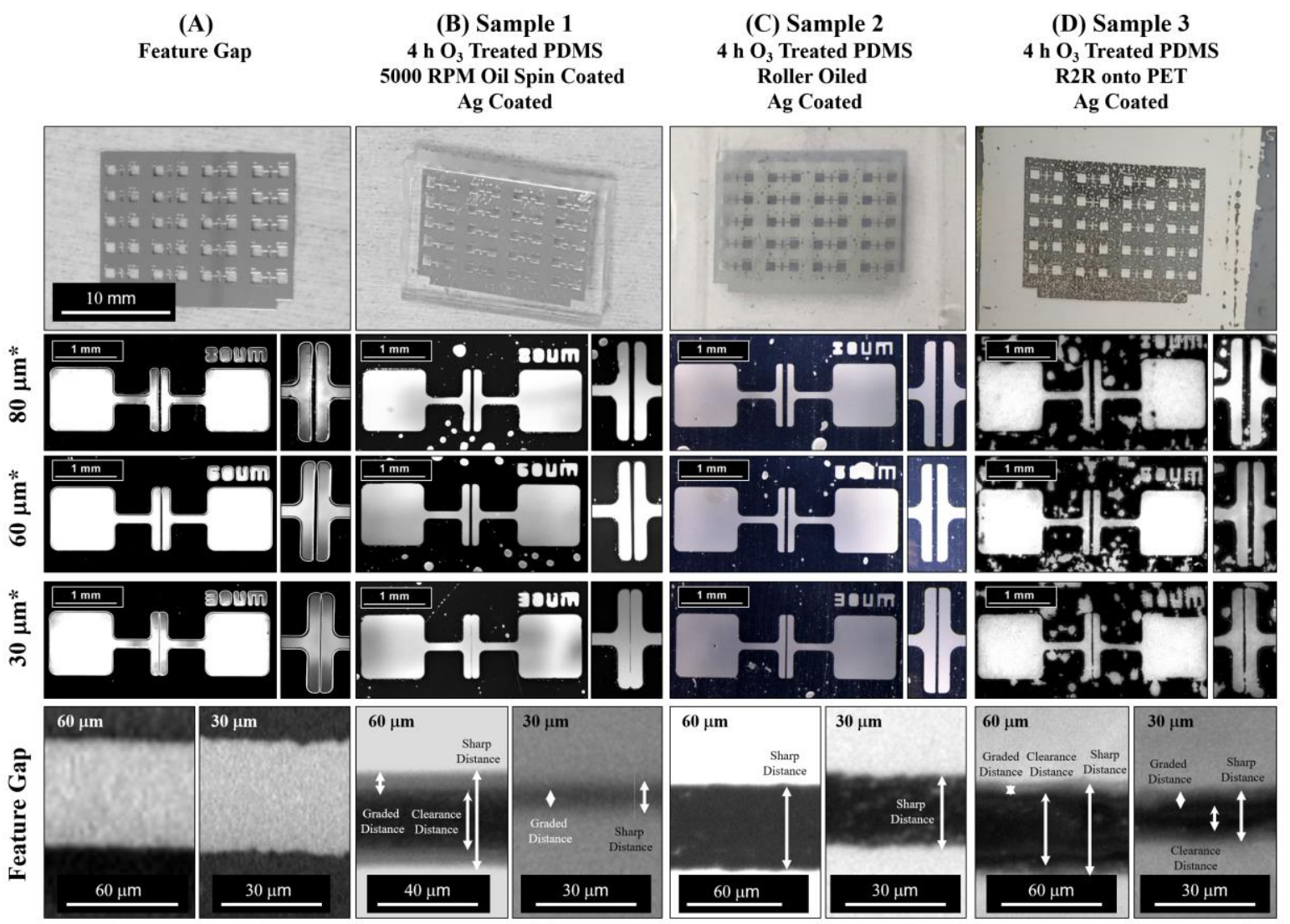

Figure 5: Micrographs of patterned samples following selective $\mathrm{O}_{3}$ treatment. *Feature channel width quoted by manufacturer, with tolerance of $\pm 7 \mu \mathrm{m}$. (A) Original mask used for selective $\mathrm{O}_{3}$ treatment (B) Ag-coated PDMS following spin coating with oil (C) Ag-coated PDMS following R2R application of oil (D) Ag-coated PET, pattern applied from PDMS stamp by $R 2 R$ transfer (specific imaging represents the $5^{\text {th }}$ compression between oiled PDMS and PET as shown in (see Figure 6C)).

\section{Sample 2 (4h $\mathrm{O}_{3}$ treated PDMS Roll-to-Roll oiled, Ag Coated)}

Oil was subsequently applied by roller-to-roller contact onto PDMS, in all cases (down to 30 $\mu \mathrm{m}$ nominal feature size), sharp separation distances and better conformity between measured mask width and deposited Ag channel width were observed, shrinking by between 1 and $11 \%$ with respect to the mask widths as shown in (Figure 5C and $7 B$ ). The sharp edges (no gradient) in this case suggests that without extra forces from spin coating or from compression due to 
transfer to another substrate, there is no graded region between the metallized and nonmetallized regions. Within the metallized region masked areas were again randomly present, caused by oil droplets transferring or forming within the untreated regions on the PDMS. We would expect the formation of such droplets to be more prevalent within large areas of metallized material, such as we have with this pattern, as the oil would tend to dewet by 'splitting' on the high contact-angle surface, rather than being able to reach and flow into the low contact angle regions, thus the scale and design of the pattern will influence the prevalence of such features. Demonstrated sharp/clearance distances were 24-74 $\mu \mathrm{m}$ vs. measured nominal gaps of 27-76 $\mu \mathrm{m}$ respectively (Figure $6 B$ ) Ag coating thickness was measured as $569 \pm 17$ $\mathrm{nm}$.
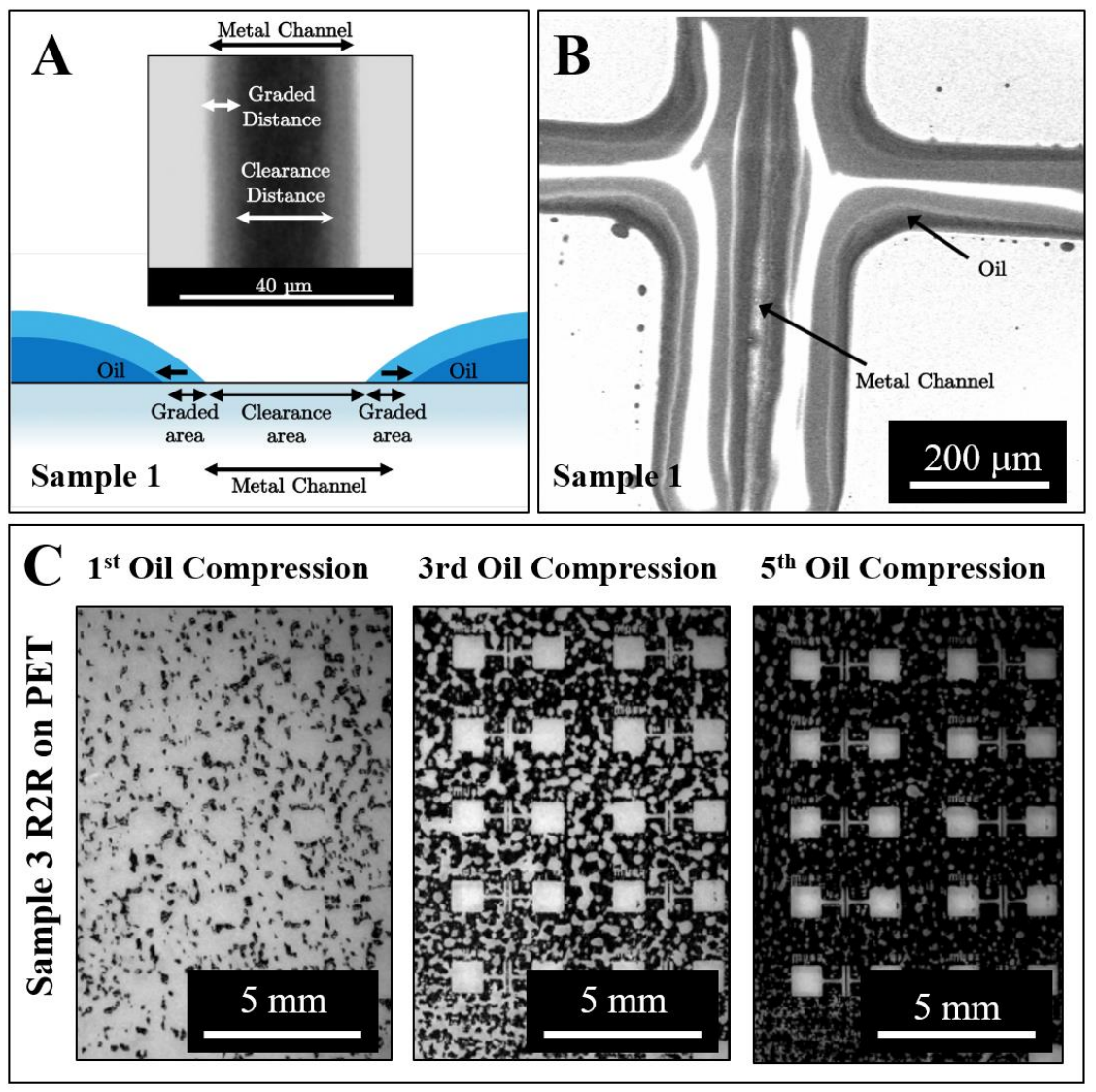

Figure 6: (A) Illustration of oil thinning towards mask edges; attributed to Ag grading as observed in samples 1 and 3. (B) Post-deposition SEM micrograph showing remaining oil constrained within the $O_{3}$ treated channel. (C) Ag-coated PET, pattern applied from PDMS stamp by R2R transfer where oil was picked up via rolling PDMS against an oiled anilox roller and subsequently rolled against the PET substrate. Successive PDMS stamping onto

PET was necessary in this case as the oil was initially too thick, spreading during compression to lead to over-oiling of the PET. Improvements were observed up to the $5^{\text {th }}$ oil application as the oil thickness on the PDMS plate was reduced (Sample 3).

\section{Sample 3 (4h $\mathrm{O}_{3}$ treated PDMS Roll-to-Roll onto PET, Ag Coated)}


As the intended application for future commercial scale processing relies of R2R oil pick up by a PDMS stamp and transfer onto a polymer substrate, the process was conducted such that oil was picked up by PDMS and transferred to PET via roller-to-roller contact followed by five+ successive stamp-to-substrate impressions. Ag coating thickness could not be directly measured using profilometry as the macro-scale roughness of the PET prevented identification of the sample edge. Patterns following PDMS to PET compression one, three and five showed gradual improvement in the developed pattern with compression one showing the consequence of over-oiling of the PDMS stamp and subsequent compression leading to oil spreading on the PET substrate. Compression five was measured for print quality - termed "Sample 3" as shown in Figure 5D. Oil quantities may be improved in future by thinning the oil layer on the anilox roller prior to transfer onto the PDMS stamp. In a continuous R2R process the stamp is re-oiled with each rotation. Sharp feature gap widths exceeded the nominal shadow mask widths by between 2-6 $\mu \mathrm{m}$ for 76 and $27 \mu \mathrm{m}$ gaps respectively whilst clearance varied from 56-14 $\mu \mathrm{m}$; an overall shrinkage of 20-49\%. In contrast to sample 2, a graded Ag distance was observed in this case in which the oil was transferred from the PDMS stamp to the PET substrate. The graded distance remained consistent between 7-12 $\mu \mathrm{m}$ attributed to liquid spreading during stamp to substrate compression (Figure 7C). 


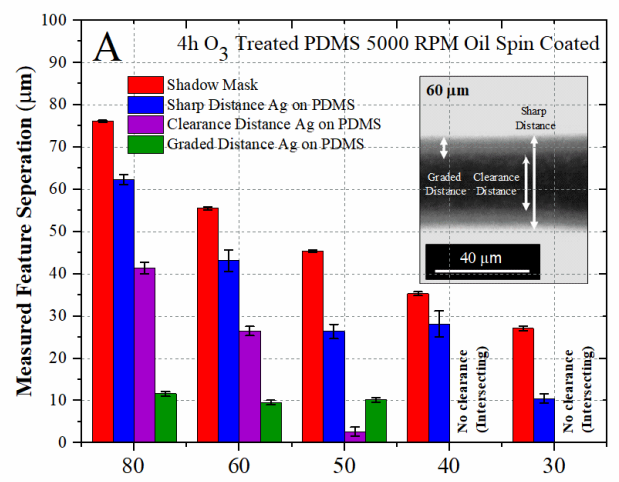

Nominal Feature Seperation Mask vs. Print $(\mu \mathrm{m})$

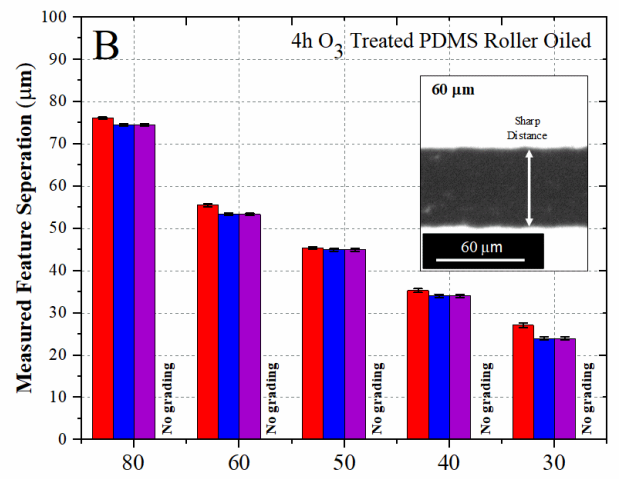

Nominal Feature Seperation Mask vs. Print $(\mu \mathrm{m})$

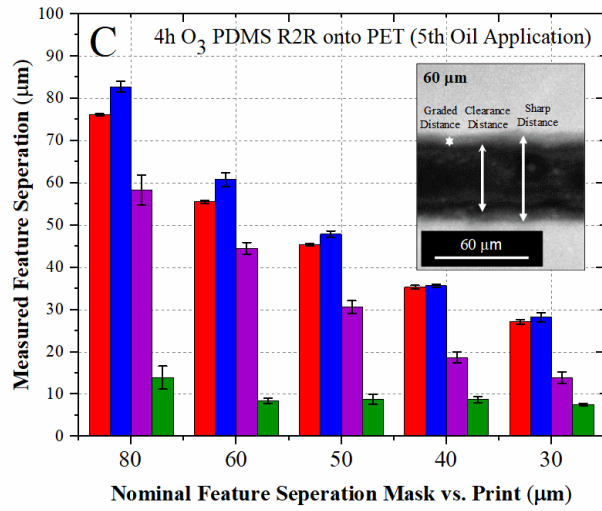

Figure 7: Measured width of Ag deposition in the controlled feature size following $4 \mathrm{~h}_{3}$ treatment compared to nominal feature size between 30-80 $\pm 7 \mu$ m. Sharp distances, Ag graded distances and gap clearance distances were measured due to the presence of Ag grading, caused by oil mask spreading for spin coated PDMS and masked PET. (A) Coated

PDMS oiled by spin coating 5000 RPM. (B). Coated PDMS oiled by R2R transfer from anilox. $(C)$ Coated PET oiled via PDMS stamp and R2R transfer from $5^{\text {th }}$ stamp to substrate compression. 


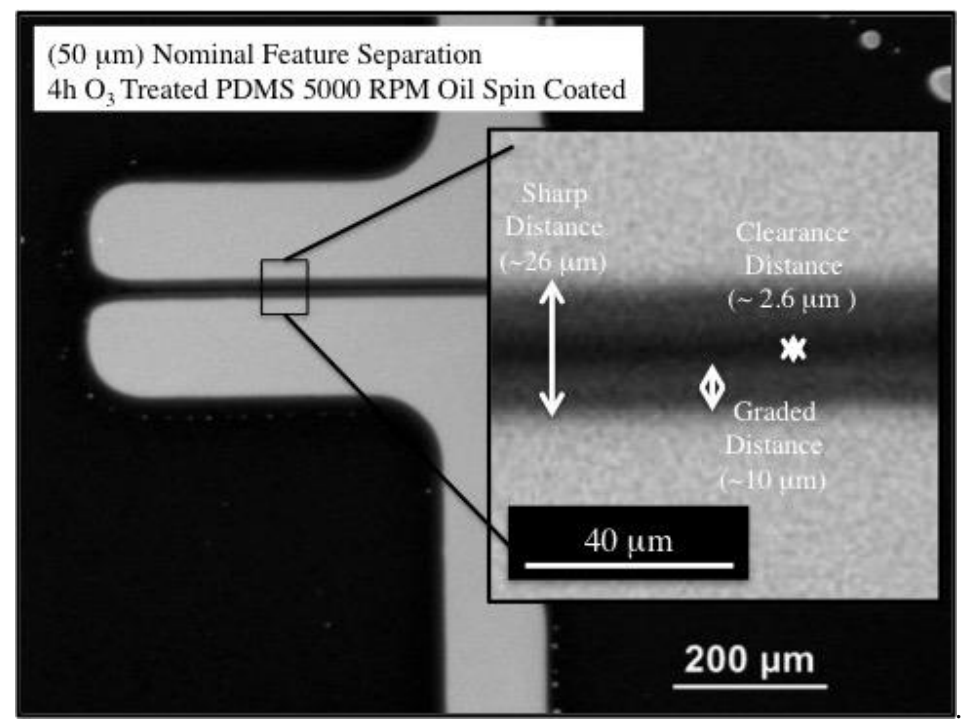

Figure 8: A minimum clearance gap of $2.6 \mu \mathrm{m}$ was identified for Ag deposited on PDMS where $50 \mu \mathrm{m}$ nominal feature separation was used as an ozone treatment mask. Smaller nominal separation, led to coalescing of deposited Ag as seen in Figure 7.

\section{Conclusions}

A mechanism has been demonstrated for selective de-wetting of Krytox ${ }^{\circledR} 1506$ oil, that can then be used within a roll-to-roll process as a liquid mask for subsequent deposition, in this case for masking metal vapors during metallization intended for future production of electrodes on flexibles. Specifically, FTIR showed that $4 \mathrm{~h} \mathrm{O}_{3}$ treatment of PDMS formed structural Si$\mathrm{OH}$ silonal groups, replacing methyl $\mathrm{Si}-\mathrm{CH}_{3}$ groups causing a reduction in contact angle from 40 to $9^{\circ}$, attributed to increase in surface energy; an effect well-documented in literature for water in microfluidic devices. PDMS stamps are typically used for micro-contact printing where patterns are based on topographical reliefs manufactured via lithography methods. As shown here, planar PDMS print plates can alternatively be manufactured by selectively altering the surface energy to manipulate liquid wetting, whilst an additional use could be to improve topographic stamps by treating raised areas such that surface forces more strongly confine the liquid. Shadow masks for selective treatment here were chosen with feature sizes in the range 27-76 $\mu \mathrm{m}$. The principle was applied to manipulate Krytox ${ }^{\circledR} 1506$ oil wetting, on elastomer and flexible substrates (PDMS and PET respectively), to demonstrate, $520 \mathrm{~nm}$ thick, Ag line widths down to $2.6 \mu \mathrm{m}$ by spin coating oil on PDMS prior to Ag deposition. Shrinkage of the feature size, as compared to the shadow mask width, was $94 \%$. In tests reproducing the steps of a continuous roll-to-roll process, rolling oil onto treated PDMS allowed $568 \mathrm{~nm}$ thick Ag with minimum line width of $24 \mu \mathrm{m}$ to be achieved; $11.6 \%$ shrinkage compared to the $27.1 \mu \mathrm{m}$ mask. A final demonstration of a full R2R application of the oil, using PDMS as a stamp to 
transfer the oiled pattern by roller to a PET substrate, produced Ag-coated PET with line widths down to $13.3 \mu \mathrm{m}$; a $48 \%$ relative shrinkage as compared to the mask. Liquid spreading is a commonly observed problem during Ink Jet printing of functional materials, resulting in increased dimensions of printed features. In contrast this spreading effect has been shown here to be beneficial given that liquid is instead used here as masking agent. By spreading, the achievable feature size of the deposited functional layer is reduced and this benefit has been best shown by up to $94 \%$ dimension reduction to reduce the mask channel width from $45.5 \mu \mathrm{m}$ to $2.6 \mu \mathrm{m}$ after deposition. The findings here show capability to produce patterned bulk metals such as Ag with feature sizes capable of challenging current state of the art ink jet technologies, with potential promise in the field of printed electronics.

\section{Acknowledgements}

This work was supported by the Engineering and Physical Sciences Research Council [grant number EP/M015173/1] via the Wearable and flexible technologies enabled by advanced thinfilm manufacture and metrology (WAFT) Collaboration. The authors would like to acknowledge Oxford Materials Characterization Services (OMCS) for equipment access and Robin Vincent and Richard Turner for Technical Assistance.

\section{References}

[1] S. Khan, L. Lorenzelli, R.S. Dahiya, Technologies for Printing Sensors and Electronics Over Large Flexible Substrates: A Review, IEEE Sensors Journal 15(6) (2015) 3164-3185.

[2] A. Larmagnac, S. Eggenberger, H. Janossy, J. Voros, Stretchable electronics based on Ag-PDMS composites, Sci Rep 4 (2014) 7254.

[3] R.V.K. Rao, K.V. Abhinav, P.S. Karthik, S.P. Singh, Conductive silver inks and their applications in printed and flexible electronics, Rsc Advances 5(95) (2015) 77760-77790.

[4] B.P. Piwcyzk, Vacuum deposition methods and masking structure, Google Patents, 1977.

[5] K. Nagatomi, T. Kajiwara, H. Ookuma, R. Tachibana, Process for producing metallized plastic film, Google Patents, 1989.

[6] T. Cosnahan, A.A. Watt, H.E. Assender, Modelling of a vacuum metallization patterning method for organic electronics, Surface and Coatings Technology 336 (2018) 128-132.

[7] X. Zhou, H. Xu, J. Cheng, N. Zhao, S.C. Chen, Flexure-based Roll-to-roll Platform: A Practical Solution for Realizing Large-area Microcontact Printing, Sci Rep 5 (2015) 10402.

[8] Y. Deng, E. Shang, Y. Liu, W. Gao, Y. Chen, C. Bao, P. Yan, J. Jiang, An overview on design of homebuilt micro-contact transfer printing machine with easy access to one micron patterning resolution, 2017 IEEE International Conference on Manipulation, Manufacturing and Measurement on the Nanoscale (3M-NANO), IEEE, 2017, pp. 242-245.

[9] X. Liu, J.T. Guthrie, A review of flexographic printing plate development, Surf Coat Int Pt B-C 86(2) (2003) 91-99.

[10] Noortec, Flexcel Direct System. <http://www.noortec.com/kodak/flexographic-andletterpress/flexcel-direct-system/\#p528>). 
[11] S. Kim, H. Sojoudi, H. Zhao, D. Mariappan, G.H. McKinley, K.K. Gleason, A.J. Hart, Ultrathin highresolution flexographic printing using nanoporous stamps, Science Advances 2(12) (2016) e1601660. [12] N.E. Stankova, P.A. Atanasov, R.G. Nikov, R.G. Nikov, N.N. Nedyalkov, T.R. Stoyanchov, N. Fukata, K.N. Kolev, E.I. Valova, J.S. Georgieva, S. Armyanov, Optical properties of polydimethylsiloxane (PDMS) during nanosecond laser processing, Applied Surface Science 374 (2016) 96-103.

[13] J. Zhou, A.V. Ellis, N.H. Voelcker, Recent developments in PDMS surface modification for microfluidic devices, Electrophoresis 31(1) (2010) 2-16.

[14] S. Hassan, M. Yusof, S. Ding, M. Maksud, M. Nodin, K. Mamat, M. Sazali, M. Rahim, Investigation of Carbon Nanotube Ink with PDMS Printing Plate on Fine Solid Lines Printed by Micro-flexographic Printing Method, IOP Conference Series: Materials Science and Engineering, IOP Publishing, 2017, p. 012017.

[15] A. Lorenz, A. Senne, J. Rohde, S. Kroh, M. Wittenberg, K. Kruger, F. Clement, D. Biro, Evaluation of Flexographic Printing Technology for Multi-Busbar Solar Cells, Enrgy Proced 67 (2015) 126-137.

[16] P.F. Moonen, I. Yakimets, J. Huskens, Fabrication of transistors on flexible substrates: from massprinting to high-resolution alternative lithography strategies, Adv Mater 24(41) (2012) 5526-41.

[17] Y. Berdichevsky, J. Khandurina, A. Guttman, Y.H. Lo, UV/ozone modification of poly(dimethylsiloxane) microfluidic channels, Sensor Actuat B-Chem 97(2-3) (2004) 402-408.

[18] D. Bodas, C. Khan-Malek, Formation of more stable hydrophilic surfaces of PDMS by plasma and chemical treatments, Microelectronic Engineering 83(4-9) (2006) 1277-1279.

[19] K. Ma, J. Rivera, G.J. Hirasaki, S.L. Biswal, Wettability control and patterning of PDMS using UVozone and water immersion, Journal of colloid and interface science 363(1) (2011) 371-378.

[20] B.W. Stuart, X. Tao, D. Gregory, H.E. Assender, Roll-to-Roll Patterning of Al/Cu/Ag Electrodes on Flexible Polyethylene by Oil Masking: A comparison of Thermal Evaporation and Magnetron Sputtering. Applied Surface Science. (2019).

[21] D.L. Williams, A.T. Kuhn, M.A. Amann, M.B. Hausinger, M.M. Konarik, E.I. Nesselrode, Computerised measurement of contact angles, Galvanotechnik 101(11) (2010) 2502.

[22] S. Wu, Calculation of interfacial tension in polymer systems, Journal of Polymer Science Part C: Polymer Symposia, Wiley Online Library, 1971, pp. 19-30.

[23] A.E. Ozcam, K. Efimenko, J. Genzer, Effect of ultraviolet/ozone treatment on the surface and bulk properties of poly(dimethyl siloxane) and poly(vinylmethyl siloxane) networks, Polymer 55(14) (2014) 3107-3119.

[24] K. Efimenko, W.E. Wallace, J. Genzer, Surface modification of Sylgard-184 poly(dimethyl siloxane) networks by ultraviolet and ultraviolet/ozone treatment, J Colloid Interface Sci 254(2) (2002) 306-15. [25] DuPont ${ }^{\mathrm{TM}} \quad$ Krytox $^{\circledR} \quad$ Performance Lubricants. $<$ http://www2.dupont.com/Lubricants/en US/assets/downloads/H58530 1.pdf>, 2003).

[26] H. Nakae, R. Inui, Y. Hirata, H. Saito, Effects of surface roughness on wettability, Acta Materialia 46(7) (1998) 2313-2318.

[27] Ossila, Source-Drain Deposition Masks for High-Density OFETs. $<$ https://www.ossila.com/products/ofet-source-drain-mask-high-density>). 